\title{
CUTANEOUS COMPLICATIONS IN MACROLIDE PRESCRIBING IN PEDIATRICS
}

\author{
Debajyoti Saha, Shatavisa Mukherjee*, Sabnam Ara Begum, Santanu Kumar Tripathi \\ Department of Clinical and Experimental Pharmacology, \\ Calcutta School of Tropical Medicine, Kolkata, India.
}

\begin{abstract}
Cutaneous adverse reactions are very common adverse effect of post antibiotic therapy. Cutaneous adverse reactions include skin lesions in the form of rashes urticaria, maculopapular rashes, erythema multiforme, Stevens Johnson syndrome and toxic epidermal necrolysis. However macrolides, generally considered safer in this regard, are frequently used to treat both adult and childhood infections, with favorable outcomes. Cutaneous manifestations of this class of antibiotic are scarce. The present case series focuses on conglomeration of few cases of cutaneous reactions due to macrolide therapy in both children and adult population.
\end{abstract}

Keywords: Macrolides, Cutaneous Adverse Drug Reactions, Acute Generalized Exanthematous Pustulosis, Maculopapular Rash.

\section{Introduction}

Macrolides, considered one of the safest options among antimicrobials, structurally have a lactone ring bonded to one or more deoxy sugar molecules. [1] Macrolides share a similar spectrum of antimicrobial activity with benzylpenicillin making them useful alternatives for people with background of penicillin and cephalosporin allergy. [2] Allergic responses to macrolides are rare, evidenced in 0.4 to $0.3 \%$ patients. [3] Adverse drug reactions (ADRs) due to macrolide therapy are generally cutaneous, with presentations ranging from urticaria/angioedema, rhinoconjunctivitis, anaphylaxis, maculopapular rash (MPR), non-immediate urticaria, contact dermatitis, fixed drug eruptions, maculopapular rash to acute generalized exanthematous pustulosis (AGEP). Severe cutaneous presentations involving necrolysis are rare.

Though macrolide hypersensitivity is not so common, literature survey lists down only a few reports of mild to moderately severe cutaneous ADRs due to this drug class, thus challenging the comprehension of underlying immune-allergenic pathophysiology. The current case series presents a cluster of few cases of skin reactions due to macrolide therapy in pediatric population.

\section{Case 1}

A 12 year old male child presented with widespread erythema and hundreds of small, flacid, confluent, nonfollicular pustules, especially on back neck along with groins and flexor aspects of legs. Reaction was observed after three days of tablet azithromycin $250 \mathrm{mg}$ once daily intake, indicated for upper respiratory tract infection (URTI). The rash was associated with mild

eISSN: 2523-6709

pISSN: 2523-6695

DOI: $10.31878 /$ ijcrpp.2019.33.1 fever, body ache and malaise, with no mucosal involvement. All laboratory investigations were within normal limits. The case was diagnosed to be azithromycin induced AGEP. The offending drug was withdrawn and he was treated conservatively with oral methyl prednisone $8 \mathrm{mg}$ (one and a half tablets for three days), levocetirizine $5 \mathrm{mg}$ once daily, topical application of calamine lotion. He responded well to the therapy and rash subsided within 3 days.

\section{Case 2}

A 5 year old male child presented with erythematous pruritic rash on his back after intake of clarithromycin syrup in dose of $125 \mathrm{mg} / 5 \mathrm{ml}$, indicated for sore throat along with moderate grade fever. Concomitantly paracetamol syrup was also advised. On the third day of treatment initiation, rash appeared at his back and medial aspect of his thigh. Laboratory investigations involving blood and liver parameters were within normal range. Clarithromycin was withdrawn and the patient was symptomatically managed with levocetirizine syrup and emollients. The rash subsided within next two days.

\section{Case 3}

A 12 year old male child presented with widespread erythema and numerous small, flaccid, confluent, nonfollicular pustules, covering the back, neck and flexor region of legs, evolving two days after being initiated on once daily dosing of clarithromycin $250 \mathrm{mg}$ for URTI. The rash was accompanied with fever, body ache and malaise. The case was diagnosed as clarithromycin induced AGEP and the drug was withdrawn. He was treated conservatively with oral methyl prednisolone 8 $\mathrm{mg}$ (one and a half tablets for three days), levocetirizine $5 \mathrm{mg}$ OD and calamine lotion and paracetamol $500 \mathrm{mg}$ as needed. He responded well to the therapy. The rash subsided slowly.

Correspondence: Ms. Shatavisa Mukherjee, Department of Clinical and Experimental Pharmacology, Calcutta School of Tropical Medicine, Kolkata, E-Mail: shatavisa100@gmail.com 


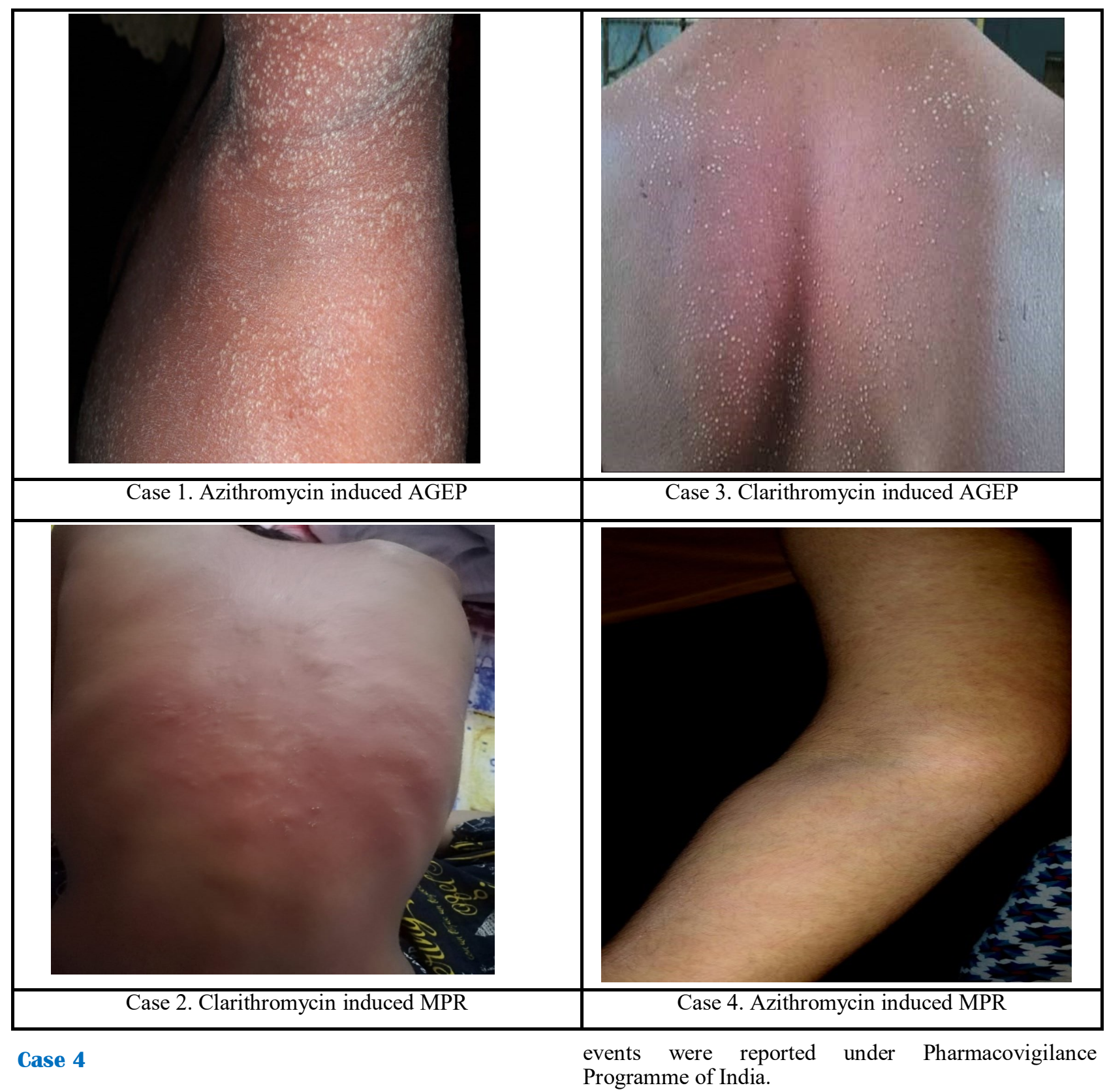

A 15 year old female child presented with erythematous maculopapular rash on consuming azithromycin $500 \mathrm{mg}$ once daily orally, indicated for sore throat. Patient reported taking azithromycin at a dose of $500 \mathrm{mg}$ once daily for two days, after which she developed rash on her upper arms. The rash examined to be pruritic, progressed to involve medial aspect of thighs and legs in a couple of days. The patient was afebrile and the rash was however not associated with urinary abnormalities or mucosal and conjunctival involvement. Laboratory investigations conferred mild decreased hemoglobin, raised eosinophils while other hemogram and liver parameters along with serum urea and creatinine within normal limits. Suspected drug was dechallenged. The patient was managed with hydroxyzine $25 \mathrm{mg}$ once daily orally along with topical application of calamine lotion as emollient to which she responded well.

Causality assessment of all the reactions conferred it to be "probable" with WHO UMC causality assessment scale, while the severity of all reactions were assessed to be moderate (level 3) as per Hartwig Seigel's Scale. The

\section{Discussion}

Cutaneous ADRs accounts for $10-30 \%$ of all ADRs, and are mostly attributed to antibiotics. Reactions can vary in severity mostly being mild-to-moderate grade though severe reactions are also estimated to occur in $0.1-2 \%$ of cases. [4] Drug allergy to antibiotics may present in the form of immediate or delayed hypersensitivity reactions. Manifestations of antibiotic allergy may be cutaneous, organ-specific, systemic or a combination of these. However allergic reactions to macrolides appear to be relatively uncommon with limited case reports of immediate and delayed reactions been reported, in both children and adults, on clarithromycin and azithromycin. In some cases successful desensitization has also been reported. [5, 6]

Despite the rampant use, pediatric safety data regarding macrolide usage is scarce in pediatric population. Examining a cutaneous ADR needs consideration of underlying bacterial infection, viral exanthems if any along with exclusion of other idiopathic plausible causes. So the clinician should negate all possibilities 
before coming into a diagnostic conclusion. Unavailability of available tests in regular set ups to diagnose drug rash creates significant challenge. Careful history taking and establishing a definite temporality is essential to establish a drug-adverse effect relationship. However rechallenge of drug for determining the relatedness of a suspected drug with the event is often a great challenge, as it may pose a potential risk of life threatening response in some cases.

Management involves identifying the putative antibiotic though a detailed medication history, along with in-vivo and in-vitro allergological investigations as feasible. Drug desensitization is considered in few situations where benefits outweigh the risks, and no alternative antibiotics can be used for various other reasons. [7] Management usually involves prompt withdrawal of the suspected drug followed by use of topical steroids for reduction of inflammatory response along with oral antihistamines and emollients for symptomatic relief. Systemic immunomodulatory drugs are also used for suppression of severe cutaneous/systemic reactions.

\section{Conclusion}

Antibiotics, one of the greatest and indispensable discoveries in medicine, are not devoid of complications. Cutaneous reactions, commonly experienced as adverse effects of post antibiotic therapy, should be cautiously managed with prompt withdrawal of the suspected agent. Avoidance of putative antibiotic through proper patient education may minimize the chance of reactions in future.

Source of Support: Nil.

Conflict of Interest: None Declared.

\section{Acknowledgement}

The authors acknowledge and support the untiring efforts and contribution of Pharmacovigilance Programme of India (PvPI) towards ensuring better patient safety nationwide.

\section{References}

[1] Dinos GP. The macrolide antibiotic renaissance. Br J Pharmacol. 2017;174(18):2967-2983.

[2] Fair RJ, Tor Y. Antibiotics and bacterial resistance in the 21 st century. Perspect Medicin Chem. 2014;6:25-64.

[3] Demoly P, Benahmed S, Valembois M, Sahla H, Messaad D, Godard P et al. Allergy to macrolide antibiotics. Review of the literature. Presse Med. 2000;29(6):321-6.

[4] Cutaneous drug reaction to antibiotics. Accessed from https://www.dermnetnz.org/topics/ cutaneous-adverse-reactions-to-antibiotics/. Accessed on May 30, 2019.

[5] Thong BY. Update on the management of antibiotic allergy. Allergy Asthma Immunol Res. 2010;2(2):77-86.

[6] Sánchez-Borges M, Thong B, Blanca M, Ensina LF, González-Díaz S, Greenberger PA et al. Hypersensitivity reactions to non beta-lactam antimicrobial agents, a statement of the WAO special committee on drug allergy. World Allergy Organ J. 2013;6(1):18.

[7] Caimmi S, Caffarelli C, Saretta F, Liotti L, Crisafulli G, Cardinale F et al. Drug desensitization in allergic children. Acta Biomed. ;90(3-S):20-29. 